\title{
Análise de campo médio para um modelo epidêmico via passeios aleatórios em um grafo
}

\author{
Renato Jacob Gava
}

\author{
DISSERTAÇÃO APRESENTADA \\ AO INSTITUTO DE MATEMÁTICA E ESTATÍSTICA \\ DA UNIVERSIDADE DE SÃO PAULO \\ PARA OBTENÇÃO \\ DO GRAU DE MESTRE EM CIÊNCIAS
}

Área de Concentração: Estatística

Orientador: Prof. Dr. Fábio Prates Machado

São Paulo, setembro de 2007.

O autor agradece ao CNPq pelo apoio financeiro (Processo N. 132646/2006-4). 


\title{
Análise de campo médio para um modelo epidêmico via passeios aleatórios em um grafo
}

\author{
Este exemplar corresponde à redação \\ final da dissertação devidamente corrigida \\ e defendida por Renato Jacob Gava \\ e aprovada pela Comissão Julgadora.
}

São Paulo, setembro de 2007.

Banca examinadora:

Prof. Dr. Fábio Prates Machado (IME-USP)

Prof. Dr. Maurício Zuluaga Martinez (UFPE)

Prof. Dr. Makoto Yoshida (UNESP) 
Para Marlene, Reinaldo e Débora 


\section{Agradecimentos}

Esta dissertação teve a colaboração de muitos, desde os amigos que me acolheram quando aqui cheguei até aqueles com quem dividi as tarefas acadêmicas. Várias foram as pessoas com quem troquei idéias e aprendi, a todas agradeço, em especial ao meu orientador Prof. Dr. Fábio Prates, pelo empenho e confiança dedicados.

Agradeço a Maurício Zuluaga, pelas sugestões que enriqueceram o trabalho e amizade.

A Paulo Marques e Fábio Lopes, que me ajudaram com os cálculos computacionais.

Aos meus pais, Reinaldo e Marlene, e a Débora, por tudo.

Aos colegas da Secretaria de Pós-graduação, pelo profissonalismo e gentilezas.

$\mathrm{E}$, finalmente, ao $\mathrm{CNPq}$, pela verba. 


\section{Resumo}

Estudamos sistemas de passeios aleatórios sobre os vértices de um grafo completo. Inicialmente há uma partícula em cada vértice do grafo das quais somente uma está ativa, as outras estão inativas. A partícula ativa realiza um passeio aleatório simples a tempo discreto com tempo de vida que depende do passado do processo, movendo-se ao longo de elos. Quando uma partícula ativa encontra uma inativa, esta se ativa; quando salta sobre um vértice já visitado, morre. O objetivo desta dissertação é estudar a cobertura do grafo completo, ou seja, a proporção de vértices visitados ao fim do processo, quando o número $n$ de vértices tende ao infinito. Analisamos as equações de campo médio para o processo descrito acima, comparando os seus resultados com os do modelo aleatório. Aqui, os resultados do campo médio parecem reproduzir os do modelo aleatório. Depois, apresentamos um estudo similar entre o modelo estocástico e as equações de campo médio para o caso em que cada partícula possui 2 vidas. Finalmente, observamos a cobertura do grafo completo para as equações de campo médio quando o número de vidas por partículas é maior que dois.

Palavras-chave: campo médio, cobertura, grafos completos, modelo dos sapos, passeios aleatórios. 


\section{Abstract}

We study random walks systems on complete graphs. Initially there is a particle at each vertex of the graph; only one is active and the other are inactive. An active particle performs a discrete-time simple random walk with lifetime depending on the past of the process moving along edges. When an active particle hits an inactive one, the latter is activated. When it jumps on a vertex which has been visited before it dies. The goal of this work is to study the coverage of the complete graph, that is, the proportion of visited vertices at the end of the process, when the number of vertex goes to infinity. We analyze the mean field equations to the process cited above, comparing their results with the ones of the random model. Here the results of the mean field approach seem to reproduce the ones of the random model. After we present a similar study between the stochastic model and mean field approximation to the case that each particle has 2 lifes. Finally we observe the coverage of the complete graph to the mean field equations when the number of lifes by particle is bigger than two.

Key words: complete graphs, coverage, frog model, mean field, random walks. 


\section{Sumário}

1 O modelo 1

1.1 Introdução . . . . . . . . . . . . . . . . . . . . . . . 1

1.2 Descrição do modelo e definições . . . . . . . . . . . . . . . . 3

1.3 Análise combinatória e computacional . . . . . . . . . . . 4

1.3.1 Uma vida por partícula . . . . . . . . . . . . . . 4

1.3.2 Duas vidas por partícula . . . . . . . . . . . . 8

2 Simulações $\quad 14$

2.1 Uma vida por partícula . . . . . . . . . . . . . . . . 14

2.2 Duas vidas por partícula . . . . . . . . . . . . . . . 15

3 Análise de campo médio $\quad 17$

3.1 Uma vida por partícula . . . . . . . . . . . . . . . . . 17

3.2 Duas vidas por partícula . . . . . . . . . . . . . . . . . . . 28

3.3 L vidas por partícula . . . . . . . . . . . . . . . . . . 35

$\begin{array}{ll}\text { Referências Bibliográficas } & 37\end{array}$ 


\section{Capítulo 1}

\section{O modelo}

\subsection{Introdução}

O modelo epidêmico abordado nesta dissertação, mais conhecido como modelo dos sapos, é parte de uma vasta área da Probabilidade. Saber como se difunde um vírus numa rede de computadores, por exemplo, é algo que desperta o interesse de probabilistas e isso exige modelagens. Isso é o que nos motiva e portanto tentamos reproduzir a difusão de um vírus na Web. Lembramos que nosso modelo é apenas mais um dentre os modelos epidêmicos e, desse modo, não visa a encerrar as buscas sobre modelos de propagação de um vírus numa rede. Aqui trabalhamos com o grafo completo, grafo no qual todos os vértices ("computadores"), um número finito deles, estão ligados entre si por elos, e o tempo de vida das partículas ("vírus") depende do passado. Inicialmente há uma partícula em cada vértice do grafo, mas somente uma está ativa, chamada raiz do processo; as outras estão inativas. Cada partícula tem a priori $L$ vidas, $L \geq 1$. Toda partícula ativa 
realiza um passeio aleatório simples independente a tempo discreto. Quando uma partícula ativa salta sobre uma inativa, esta se ativa; quando salta sobre um vértice já visitado, perde uma de suas $L$ vidas. Uma partícula ativa se move até não lhe restarem vidas. E essa é uma diferença importante deste modelo para modelos nos quais cada partícula possui uma probabilidade $p$ de sobreviver e $1-p$ de morrer a cada instante para depois possivelmente saltar, como podemos ver em [1], [2], [5].

Antes de [3] não se conhece referências sobre este tipo de modelo, artigo no qual baseia-se esta dissertação. Em [7] pode-se encontrar a versão dele a tempo contínuo, com a variação de que só uma partícula salta por vez e o tempo de espera de cada salto tem distribuição exponencial, e a demonstração para este caso de um indício que também temos aqui: conforme cresce o número de vértices as trajetórias do conjunto de vértices visitados e do conjunto de partículas ativas a cada instante convergem para trajetórias determinísticas.

A finalidade deste trabalho é estudar a cobertura do grafo completo, ou seja, a proporção de vértices visitados ao término do processo, quando o número $n$ de vértices tende ao infinito. Limitamo-nos aos casos em que $L=1$ e $L=2$. Para isso, avaliamos no capítulo 3 as equações de campo médio para o processo descrito acima, comparando os seus resultados com os obtidos por simulação, os quais podem ser vistos no capítulo 2. Os resultados do campo médio são condizentes com os do modelo aleatório. Antes, entretanto, no capítulo 1, apresentamos a 
descrição detalhada do modelo e sua análise combinatória.

\subsection{Descrição do modelo e definições}

Seja $K_{n}$ o grafo completo de $n$ vértices, ou seja, cada vértice está conectado a todos os outros por meio de elos. Seja $L \geq 1$ um número inteiro fixo. Poremos $K_{n}$ uma dinâmica que evolui a tempo discreto: no instante inicial temos uma partícula em cada vértice, todas com $L$ vidas, porém somente uma está ativa. Todas as outras estão inativas.

Toda partícula ativa realiza um passeio aleatório simples a tempo discreto e escolhe uniformente um vértice sobre o qual salta; se a partícula originalmente colocada aí está inativa, então ambas (ou todas as que saltarem neste vértice) tornam-se ativas, caso contrário a partícula que pula (ou todas as que aí pularem) perde uma das vidas restantes. Cada partícula ativa tem trajetória independente e se move até perder as todas vidas.

Definimos agora, respectivamente, $A_{t}, I_{t}, D_{t}$ como o número de partículas ativas, o número de partículas inativas e o número de partículas mortas no tempo t. $A_{t}+I_{t}+D_{t}=n$ para todo $t$. Da mesma forma, $A_{t}^{i}, i=1, \ldots, L$, corresponde ao número de partículas ativas com $i$ vidas no tempo $t$, com $A_{t}=\sum_{i=1}^{L} A_{t}^{i}$, e $V_{t}=A_{t}+D_{t}$ ao número de vértices visitados no tempo $t$. Em geral, tomamos $A_{0}=2=A_{0}^{L}, A_{0}^{i}=0, i=1, \ldots, L-1$, e $D_{0}=0$, já que após o primeiro instante sempre existem duas partículas ativas e com $L$ vidas. 
Objetivamos estudar a proporção de vértices visitados ao final do processo quando o número $n$ de vértices tende ao infinito. Assim, consideramos o modelo de campo médio referente ao processo descrito acima para $L=1$ e $L=2$, confrontando os resultados de ambos os modelos. As equações de campo médio são uma aproximação da análise do processo, já que descrevem o comportamento dele por meio de médias e não consideram as correlações que nele existem, além de não observarem o deslocamento de cada partícula ativa.

Tais equações, apresentadas no capítulo 3, como no modelo original, têm as seguintes características: $a_{t}, i_{t}, d_{t}$ representam, respectivamente, a proporção de partículas ativas, a proporção de partículas inativas e a proporção de partículas mortas no tempo $t . a_{t}+i_{t}+d_{t}=1$ para todo $t$. Similarmente, $a_{t}^{i}, i=1, \ldots, L$, corresponde à proporção de partículas ativas com $i$ vidas no tempo $t, \operatorname{com} a_{t}=$

$\sum_{i=1}^{L} a_{t}^{i}$, e $v_{t}=a_{t}+d_{t}$ à proporção de vértices visitados no tempo $t$. Usualmente, tomamos $a_{0}=2 / n=a_{0}^{L}, a_{0}^{i}=0, i=1, \ldots, L-1$, e $d_{0}=0$, já que após o primeiro instante sempre existem duas partículas ativas e com $L$ vidas.

\subsection{Análise combinatória e computacional}

\subsubsection{Uma vida por partícula}

Seja $(A=a, I=i, D=d)$ ou $(a, i, d)$ um possível estado deste modelo que nos diz o número de partículas ativas, inativas e mortas, respectivamente. Alguns fatos desta dinâmica são:o número de partículas mortas cresce, o número 
de partículas inativas decresce, $a+i+d=n$, cada estado possível só é atingido no máximo uma vez, o número de partículas ativas pode no máximo dobrar de um instante para outro, o número mínimo de partículas ativas, se as temos, é duas.

Observemos então que não é possível passar de um estado da forma $\left(a^{\prime}, i, d^{\prime}\right)$ para um estado da forma $(a, i, d)$ (mesmo número de partículas inativas) se $a \neq$ 0, pois não acordar nenhuma partícula significa que todas as partículas ativas pularam sobre sitíos cujas partículas originais já foram acordadas e portanto todas morreriam.

Seja $P$ a medida de probabilidade deste processo. Então as condições iniciais são: $P(2, n-2,0)=1, P(1, n-2,1)=0, P(0,0, d)=0$. Também

$$
P(a, i, d)=\sum P\left(a^{\prime}, i^{\prime}, d^{\prime}\right) P\left(\text { passar de }\left(a^{\prime}, i^{\prime}, d^{\prime}\right) \operatorname{para}(a, i, d) \mid\left(a^{\prime}, i^{\prime}, d^{\prime}\right)\right),
$$

onde a soma é sobre os estados $\left(a^{\prime}, i^{\prime}, d^{\prime}\right)$ que podem passar para o estado $(a, i, d)$.

Notemos, pelo descrito acima, que para passar do estado $\left(a^{\prime}, i^{\prime}, d^{\prime}\right)$ para o estado $(a, i, d)(a \neq 0)$ precisamos de que $d^{\prime} \leq d, i^{\prime}>i$ e $a+i+d=a^{\prime}+i^{\prime}+d^{\prime}$ o que equivalentemente corresponde a $0 \leq d^{\prime} \leq d, i^{\prime}=i+j, j \in\{1,2, \ldots, n\}$ e $a^{\prime}=(a-j)+\left(d-d^{\prime}\right)$. Das $a^{\prime}$ partículas ativas $d-d^{\prime}$ morrerão $\left(d^{\prime}+\left(d-d^{\prime}\right)=d ! !\right)$ e $a-j$ acordarão $j$ das $i+j$ partículas inativas $((a-j)+j=a$ !!). Como tinhamos $i+j$ partículas inativas e acordamos $j$, ficamos com $i$ partículas inativas.

Inicialmente apresentamos o lema abaixo, que será útil nos teoremas que seguem. As $s$ bolas distinguíveis do enunciado fazem o papel das partículas ativas 
que saltam sobre o conjunto de partículas inativas e as $j$ urnas, das partículas inativas que serão acordadas.

Lema 1.1 Se queremos colocar s bolas distinguíveis em $j$ urnas o número de maneiras diferentes de fazer-se isto é:

$$
f(s, j):=\sum_{r=0}^{j-1}(-1)^{r}\left(\begin{array}{l}
j \\
r
\end{array}\right)(j-r)^{s}
$$

Prova : Conforme Feller (1968), seja $A_{k}$ o evento de que a $k$-ésima urna esteja vazia, $k=1, \ldots, j$. Sejam $A=A_{1} A_{2} \cup \ldots \cup A_{j}$ e $p_{i}=P\left(A_{i}\right), p_{i j}=P\left(A_{i} A_{j}\right)$, $p_{i j k}=P\left(A_{i} A_{j} A_{k}\right), \ldots$, onde o ordem dos índices não importa e dois índices nunca têm o mesmo valor. Definimos $S_{1}=\sum p_{i}, S_{2}=\sum p_{i j}, S_{3}=\sum p_{i j k}$, ... Então $P(A)=S_{1}-S_{2}+S_{3}-S_{4}+\ldots \pm S_{j}$, pelo teorema da união de eventos. Agora encontremos os valores de $S_{i}$ : admitindo que a cada arranjo está associada à probabilidade $j^{-s}$, deseja-se encontrar a probabilidade $p_{m}(s, j)$ de termos exatamente $m$ compartimentos vazios. Para uma realização de $A_{k}$ existem $(j-1)^{s}$ maneiras distintas de ocorrer. Similarmente, para se deixar duas urnas prefixadas vazias temos $(j-2)^{s}$ formas, etc. $\operatorname{Logo} p_{i}=(1-1 / j)^{s}, p_{i j}=(1-2 / j)^{s}$, ... e

$$
S_{q}=\left(\begin{array}{l}
j \\
q
\end{array}\right)\left(1-\frac{q}{j}\right)^{s}
$$

para $q \leq j$. Então

$$
p_{0}(s, j)=1-S_{1}+S_{2}-\ldots=\sum_{r=0}^{j-1}(-1)^{r}\left(\begin{array}{l}
j \\
r
\end{array}\right)\left(1-\frac{r}{j}\right)^{s}
$$


Agora mostramos as fórmulas para qualquer possível estado.

\section{Lema 1.2}

$$
P(a, i, 0)=\sum_{j=1}^{[a / 2]}\left(\begin{array}{c}
i+j \\
i
\end{array}\right)\left(\frac{1}{n-1}\right)^{a-j} f(a-j, j) P(a-j, i+j, 0) .
$$

Prova : Do estado $\left(a^{\prime}, i^{\prime}, d^{\prime}\right)$ podemos passar para o estado $(a, i, 0)$ se, e somente se, $d^{\prime}=0, i^{\prime}=i+j, a^{\prime}=a-j$ e $a-j$ partículas ativas acordam $j(f(a-j, j))$ das $i+j\left(\left(\begin{array}{c}i+j \\ i\end{array}\right)=\left(\begin{array}{c}i+j \\ j\end{array}\right)\right)$ partículas inativas. $\mathrm{E} \frac{1}{n-1}$ corresponde à probabilidade de uma partícula ativa acordar uma partícula inativa fixada. Além disso, $1 \leq j \leq[a / 2]$, pois se $j>[a / 2]$, então $2 a^{\prime}<a$, mas $2 a^{\prime} \geq a$, onde $[x]$ denota a parte inteira de $x$.

\section{Teorema 1.3}

$$
\begin{aligned}
P(a, i, d)= & \sum_{d^{\prime}=0}^{d} \sum_{j=1}^{[a / 2]}\left(\begin{array}{c}
d-d^{\prime}+a-j \\
d-d^{\prime}
\end{array}\right)\left(\begin{array}{c}
i+j \\
i
\end{array}\right)\left(\frac{1}{n-1}\right)^{d-d^{\prime}+a-j} \\
& (d+a-j-1)^{d-d^{\prime}} f(a-j, j) P\left(a-j+d-d^{\prime}, i+j, d^{\prime}\right) .
\end{aligned}
$$

Prova : Do estado $\left(a^{\prime}, i^{\prime}, d^{\prime}\right)$ podemos passar para o estado $(a, i, d)$ se, e somente se, $0 \leq d^{\prime} \leq d, i^{\prime}=i+j, a^{\prime}=(a-j)+\left(d-d^{\prime}\right)$ e das $(a-j)+\left(d-d^{\prime}\right)$ partículas ativas morrem $d-d^{\prime}\left(\left(\begin{array}{c}d-d^{\prime}+a-j \\ d-d^{\prime}\end{array}\right)\right)$ partículas; $\frac{d+a-j-1}{n-1}$ é a probabilidade de uma partícula ativa morrer, as $a-j$ que permanecem ativas acordam $j$ das $i+j$ partículas ativas, e $\frac{1}{n-1}$ é a probabilidade de uma partícula ativa acordar uma 
inativa fixada. Mais ainda: $1 \leq j \leq[a / 2]$, pois se $j>[a / 2]$, então $2(a-j)<a$, mas $2(a-j)$ é o número máximo de partículas que podem ficar ativas.

\section{Teorema 1.4}

$$
P(0, i, d)=\sum_{a=2}^{d}\left(\frac{d-1}{n-1}\right)^{a} P(a, i, d-a) .
$$

Prova : Do estado $\left(a^{\prime}, i^{\prime}, d^{\prime}\right)$ podemos passar para o estado $(0, i, d)$ se, e somente se, $d=d^{\prime}+a^{\prime}$ e $i=i^{\prime}$, isto é, se todas as partículas ativas morrem. Observemos que $\frac{d-1}{n-1}$ é a probabilidade de uma partícula ativa morrer e que o número mínimo de partículas ativas é 2 .

Notemos que a probabilidade de cobrir totalmente $K_{n}$ é dada por

$$
\rho_{n}=1-\sum_{d=2}^{n-1} P(0, n-d, d) .
$$

Usando as fórmulas anteriores computamos valores de $\rho_{n}$ para vários valores de $n \leq 200$. Os valores são apresentados na tabela 1.1, que pode ser encontrada em [3]. Conjecturamos que $\rho_{n} \rightarrow 0$ quando $n \rightarrow \infty$.

\subsubsection{Duas vidas por partícula}

Seja $\left(A_{1}=a_{1}, A_{2}=a_{2}, I=i, D=d\right)$ ou $\left(a_{1}, a_{2}, i, d\right)$ um possível estado deste modelo que nos diz o número de partículas ativas com uma vida, ativas com duas vidas, inativas e mortas, respectivamente. Fatos próprios desta dinâmica são: o número de partículas mortas cresce, o número de partículas inativas decresce, 


\begin{tabular}{|c|c|}
\hline$n$ & $\rho_{n}$ \\
\hline 10 & $2,38561 \times 10^{-1}$ \\
25 & $2,43941 \times 10^{-2}$ \\
50 & $5,36173 \times 10^{-4}$ \\
75 & $1,17556 \times 10^{-5}$ \\
100 & $2,57646 \times 10^{-7}$ \\
200 & $5,95080 \times 10^{-14}$ \\
\hline
\end{tabular}

Tabela 1.1: Valores de $\rho_{n}$.

$a_{1}+a_{2}+i+d=n$, cada estado possível só é atingido no máximo uma vez, o número de partículas ativas $a_{1}+a_{2}=a$ pode no máximo dobrar de um instante para outro.

Temos aqui pequenas diferenças em relação ao modelo anterior: é possível passar de um estado da forma $\left(a_{1}^{\prime}, a_{2}^{\prime}, i, d^{\prime}\right)$ para um estado da forma $\left(a_{1}, a_{2}, i, d\right)$ (mesmo número de partículas inativas) desde que $a_{2}^{\prime} \neq 0$, pois não acordar nenhuma partícula significa que todas as partículas ativas pularam sobre sitíos já visitados e perderam uma vida. Daí, $d=d^{\prime}+a_{1}^{\prime}, a_{1}=a_{2}^{\prime}$, o que garante-nos que $a=a_{1}+a_{2} \neq 0$.

Seja $P$ a medida de probabilidade deste processo. Então as condições iniciais são: $P(0,2, n-2,0)=1, P(0,1, n-2,1)=P(1,0, n-2,1)=P(1,1, n-2,0)=0$, $P(1,0, n-1,0)=P(0,0, n-1,1)=0$ e $P(0,1, n-d-1, d)=0$. Também $P\left(a_{1}, a_{2}, i, d\right)=\sum P\left(a_{1}^{\prime}, a_{2}^{\prime}, i^{\prime}, d^{\prime}\right) P\left(\left(a_{1}^{\prime}, a_{2}^{\prime}, i^{\prime}, d^{\prime}\right) \rightarrow\left(a_{1}, a_{2}, i, d\right) \mid\left(a_{1}, a_{2}^{\prime}, i^{\prime}, d^{\prime}\right)\right)$

onde o somatório é sobre os estados $\left(a_{1}^{\prime}, a_{2}^{\prime}, i^{\prime}, d^{\prime}\right)$ que podem passar para o estado 
$\left(a_{1}, a_{2}, i, d\right)$.

Notemos, conforme exposto acima, que para passar do estado $\left(a_{1}^{\prime}, a_{2}^{\prime}, i^{\prime}, d^{\prime}\right)$ para o estado $\left(a_{1}, a_{2}, i, d\right)(a \neq 0)$ precisamos de que $d^{\prime} \leq d, i^{\prime} \geq i$ e $a_{1}+a_{2}+$ $i+d=a_{1}^{\prime}+a_{2}^{\prime}+i^{\prime}+d^{\prime}$, o que equivalentemente corresponde a $0 \leq d^{\prime} \leq d$, $i^{\prime}=i+j, j \in\{0,1,2, \ldots, n\}$ e $a_{1}^{\prime}=\left(a+a_{2}^{\prime}-j\right)+\left(d-d^{\prime}\right)$. Das $a_{1}^{\prime}$ partículas ativas com uma vida $d-d^{\prime}$ morrerão $\left(d^{\prime}+\left(d-d^{\prime}\right)=d\right.$ !); das $a_{2}^{\prime}$ partículas ativas com duas vidas $k$ delas perderão uma vida. Logo, $a^{\prime}-\left(d-d^{\prime}\right)-k$ acordarão $j$ das $i+j$ partículas inativas $\left(\left(a^{\prime}-\left(d-d^{\prime}\right)+j=a ! !\right)\right.$. Como tinhamos $i+j$ partículas inativas e acordamos $j$, ficamos com $i$ partículas inativas. Ademais, $a_{2}=\left(a_{2}^{\prime}-k\right)+j$ e $a_{1}=a_{1}^{\prime}-\left(d-d^{\prime}\right)+k$.

Apresentamos neste momento as fórmulas para qualquer possível estado, admitindo aqui que $f(s, j)=0$ quando $s<j$ para facilitar a escrita da expressão.

\section{Lema 1.5}

$$
\begin{aligned}
P\left(a_{1}, a_{2}, i, 0\right)= & \sum_{j=1}^{[a / 2]} \sum_{k=0}^{a_{1}}\left(\begin{array}{c}
a_{2}+k-j \\
k
\end{array}\right)\left(\begin{array}{c}
i+j \\
i
\end{array}\right)\left(\frac{1}{n-1}\right)^{a-j-k} \\
& \left(\frac{a-j-1}{n-1}\right)^{k} f(a-j-k, j) P\left(a_{1}-k, a_{2}+k-j, i+j, 0\right),
\end{aligned}
$$

onde $a_{2}>0$.

Prova : Passamos do estado $\left(a_{1}^{\prime}, a_{2}^{\prime}, i^{\prime}, d^{\prime}\right)$ para o estado $\left(a_{1}, a_{2}, i, 0\right)$ se, e somente se, $d^{\prime}=0, i^{\prime}=i+j, a^{\prime}=a-j, k$ das $a_{2}^{\prime}$ perdem uma vida $\left(\left(\begin{array}{c}a_{2}+k-j \\ k\end{array}\right)\right)$, $0 \leq k \leq a_{2}^{\prime}$, e $a_{1}^{\prime}+a_{2}^{\prime}-k=a_{1}+a_{2}-j-k$ partículas ativas acordam $j(f(a-j-k, j))$ das $i+j\left(\left(\begin{array}{c}i+j \\ i\end{array}\right)=\left(\begin{array}{c}i+j \\ j\end{array}\right)\right)$ partículas inativas, onde $a_{1}^{\prime}=a_{1}-k$ e $a_{2}^{\prime}=a_{2}+k-j$. 
$\mathrm{E} \frac{1}{n-1}$ corresponde à probabilidade de uma partícula ativa acordar uma partícula inativa fixada e $1-\frac{i^{\prime}}{n-1}=a-j-1 / n-1$ à probabilidade de uma partícula ativa perder uma vida. Ademais, $j \leq[a / 2]$, pois se $j>[a / 2]$, então $2 a^{\prime}<a$, mas $2 a^{\prime} \geq a$. E $1 \leq j$, pois se $j=0$, então $a_{2}=0$.

\section{Lema 1.6}

$$
P\left(a_{1}, 0, i, d\right)=\sum_{d^{\prime}=0}^{d}\left(\frac{a_{1}+d-1}{n-1}\right)^{a_{1}+d-d^{\prime}} P\left(d-d^{\prime}, a_{1}, i, d^{\prime}\right)
$$

onde $d \geq 0$.

Prova : Do estado $\left(a_{1}^{\prime}, a_{2}^{\prime}, i^{\prime}, d^{\prime}\right)$ podemos passar para o estado $\left(a_{1}, 0, i, d\right)$ se, e somente se, $d^{\prime} \leq d, j=0$, já que $a_{2}=0$, e $a_{1}=k=a_{2}^{\prime}$, pois todas as partículas ativas perderam uma vida. E $a_{1}+d-1 / n-1$ é probabilidade de uma partícula ativa perder uma vida.

\section{Teorema 1.7}

$$
\begin{aligned}
P\left(a_{1}, a_{2}, i, d\right)= & \sum_{d^{\prime}=0}^{d} \sum_{j=1}^{[a / 2]} \sum_{k=0}^{a_{1}}\left(\begin{array}{c}
d-d^{\prime}+a_{1}-k \\
d-d^{\prime}
\end{array}\right)\left(\begin{array}{c}
a_{2}+k-j \\
k
\end{array}\right)\left(\begin{array}{c}
i+j \\
i
\end{array}\right) \\
& \left(\frac{1}{n-1}\right)^{a-j-k}\left(\frac{d+a-j-1}{n-1}\right)^{k+d-d^{\prime}} \\
& f(a-j-k, j) P\left(a_{1}-k+d-d^{\prime}, a_{2}+k-j, i+j, d^{\prime}\right),
\end{aligned}
$$

onde $a_{2}>0$.

Prova : Passamos do estado $\left(a_{1}^{\prime}, a_{2}^{\prime}, i^{\prime}, d^{\prime}\right)$ para o estado $\left(a_{1}, a_{2}, i, d\right)$ se, e somente se, $0 \leq d^{\prime} \leq d, i^{\prime}=i+j, a_{1}^{\prime}+a_{2}^{\prime}=a_{1}+a_{2}-j+\left(d-d^{\prime}\right), k$ das $a_{2}^{\prime}=a_{2}+k-j$ 
perdem uma vida $\left(\left(\begin{array}{c}a_{2}+k-j \\ k\end{array}\right)\right), 0 \leq k \leq a_{2}^{\prime}$, das $a_{1}^{\prime}=a_{1}-k+\left(d-d^{\prime}\right)$ partículas ativas com uma vida $d-d^{\prime}$ morrem $\left(\left(\begin{array}{c}a_{1}-k+\left(d-d^{\prime}\right) \\ d-d^{\prime}\end{array}\right)\right) ; \frac{d+a-j-1}{n-1}=1-\frac{i^{\prime}}{n-1}$ é a probabilidade de uma partícula ativa perder uma vida, $a_{1}^{\prime}+a_{2}^{\prime}-\left(d-d^{\prime}\right)-k=a_{1}+a_{2}-j-k$ partículas ativas acordam $j$ das $i+j$ partículas ativas e $\frac{1}{n-1}$ é a probabilidade de uma partícula ativa acordar uma inativa fixada. Mais ainda: $1 \leq j \leq[a / 2]$, pois se $j>[a / 2]$, então $2(a-j)<a$, mas $2(a-j)$ é o número máximo de partículas que podem ficar ativas, e se $j=0$, então $a_{2}=0$.

\section{Teorema 1.8}

$$
P(0,0, i, d)=\sum_{d^{\prime}=0}^{d-1}\left(\frac{d-1}{n-1}\right)^{d-d^{\prime}} P\left(d-d^{\prime}, 0, i, d^{\prime}\right)
$$

Prova : Do estado $\left(a_{1}^{\prime}, a_{2}^{\prime}, i^{\prime}, d^{\prime}\right)$ podemos passar para o estado $(0,0, i, d)$ se, e somente se, $i=i^{\prime}, a_{2}^{\prime}=k=a_{1}=0$ e $d=d^{\prime}+a_{1}^{\prime}$, pois todas as partículas ativas perdem uma vida. Observemos que $\frac{d-1}{n-1}$ é a probabilidade de uma partícula ativa morrer e que o número mínimo de partículas ativas neste caso é 1 .

A probabilidade de cobrir totalmente o grafo $K_{n}$ é dada por

$$
\rho_{n}=1-\sum_{d=2}^{n-1} P(0,0, n-d, d)
$$

Com as expressões matemáticas e as condições de contorno dadas acima calculamos valores de $\rho_{n}$ para vários valores de $n \leq 75$. Os valores são apresentados na tabela 1.2. Pelo comportamento de $\rho_{n}$, que nos dá sinais de que decai exponencialmente, conjecturamos que $\rho_{n} \rightarrow 0$ quando $n \rightarrow \infty$. 


\begin{tabular}{|c|c|}
\hline$n$ & $\rho_{n}$ \\
\hline 5 & 0,845888 \\
6 & 0,807024 \\
7 & 0,770539 \\
8 & 0,736093 \\
9 & 0,703361 \\
10 & 0,672157 \\
25 & 0,340764 \\
50 & 0,109927 \\
75 & 0,035467 \\
\hline
\end{tabular}

Tabela 1.2: Valores de $\rho_{n}$. 


\section{Capítulo 2}

\section{Simulações}

\subsection{Uma vida por partícula}

Realizamos simulações do processo com uma vida por partícúla $n=500$, 1000,2000 e 5000 e notamos os valores de $a / n$ e $(m+a) / n$ em cada instante de tempo. Observamos que o comportamento do processo se altera pouco quando comparamos os valores alcançados pelas simulações para diferentes valores de $n$ (figura 2.1). Nosso trabalho leva-nos a acreditar que os máximos de $a / n$ e $(d+a) / n$ estão perto de 0,35 e 0,83 , respectivamente. Outro fato observado nos diz que até um instante um pouco além do máximo de $a / n$ o crescimento de $(d+a) / n$ é rapido, pois há ainda um conjunto substancial de vértices inativos, mas alguns instantes após isso $(d+a) / n$ cresce muito devagar até atingir um valor próximo a 0,83 . Há sinais de que $a / n$ e $(d+a) / n$ convirjam em distribução quando $n$ cresce, já que seus gráficos se deslocam para a direita. 

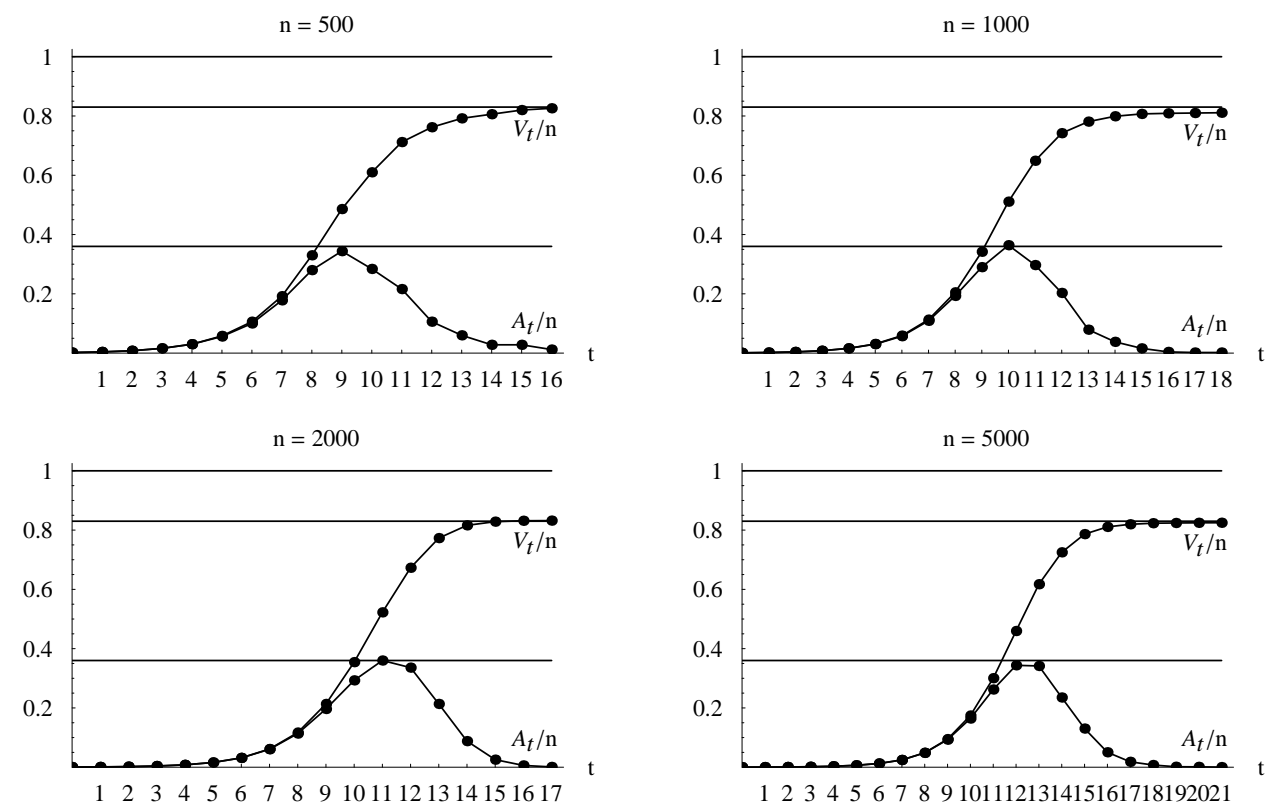

Figura 2.1: Evolução de $a / n$ e $(d+a) / n$ em simulações para $n=500,1000,2000$ e 5000. Linhas de 0,36 e 0,83 são indicadas.

\subsection{Duas vidas por partícula}

As simulações do modelo estocástico com duas vidas por partícúla foram realizadas para $n=500,1000,2000$ e 5000 e notamos os valores de $a / n$ e $(m+a) / n$ em cada instante de tempo. Observamos de novo que o comportamento do processo se altera pouco quando comparamos os valores alcançados pelas simulações para diferentes valores de $n$ (figura 2.2). Cremos que os máximos de $a / n$ e $(d+a) / n$ estão próximos a 0,63 e 0,95, respectivamente. Aqui também notamos que até um pouco além do máximo de $a / n$ o crescimento de $(d+a) / n$ é rapido, pois há ainda uma proporção significativa de vértices inativos, mas alguns instantes após isso $(d+a) / n$ cresce muito devagar até atingir um valor próximo a 0,95 . As- 
sim como para processo em que $L=1$, em virtude do deslocamento dos gráficos para a direita quando $n$ cresce, há indícios de que $a / n$ e $(d+a) / n$ convirjam em distribução.
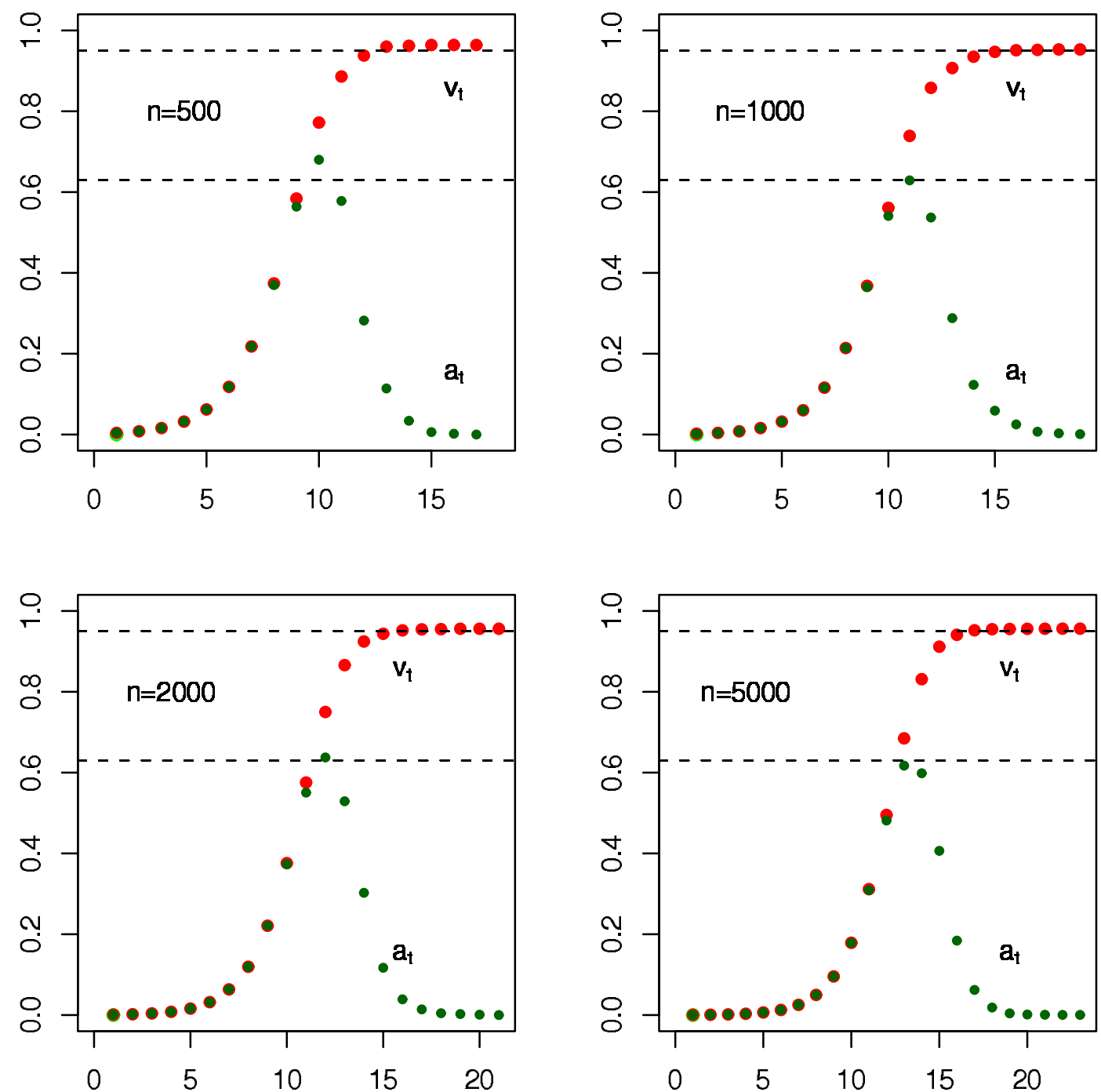

Figura 2.2: Evolução de $a / n$ e $(d+a) / n$ em simulações para $n=500,1000,2000$ e 5000. Linhas de 0,63 e 0,95 são indicadas. 


\section{Capítulo 3}

\section{Análise de campo médio}

\subsection{Uma vida por partícula}

Analisemos a dinâmica em $K_{n}$ sob a ótica da análise de campo médio, a qual não leva em conta a dependência entre as trajetórias das partículas e narra o desenvolvimento dessa dinâmica através de médias. A forma como definiremos a proporção de partículas ativas, inativas e mortas está totalmente motivada pelo que aconteceria no grafo completo se tivessemos independência. Seja $a_{0} \in$ $(0,1)$ pequeno em $t=0$; cada partícula inativa acorda com probabilidade $a_{0}$ independente das outras e definimos $d_{0}=0$. Logo em $t=0$ acordam em média $a_{0} n$ partículas. Agora definimos a dinâmica para $t+1$ :

1. Cada partícula ativa pode morrer ou pode sobreviver, morre com probabilidade $d_{t}+a_{t}$ independente das outras e sobrevive com probabilidade $1-d_{t}-a_{t}$ independente das outras, onde $d_{t}$ e $a_{t}$ são a proporção de partículas mortas e ativas no instante $t$, respectivamente. Logo morrem em 
média $\left(d_{t}+a_{t}\right) a_{t} n$ partículas e sobrevivem em média $\left(1-d_{t}-a_{t}\right) a_{t} n$ partículas.

2. Cada partícula inativa pode acordar ou não, acorda com probabilidade $\left(1-\exp \left(-a_{t}\right)\right)$ independente das outras. Logo acordam em média $\left(1-d_{t}-a_{t}\right) n\left(1-\exp \left(-a_{t}\right)\right)$ partículas. Observe que $\left(1-d_{t}-a_{t}\right)$ é a proporção de partículas inativas no instante $t$ e $\left(1-d_{t}-a_{t}\right) n$ é o número de partículas inativas no instante $t$.

Vejamos a motivação: seja $(a, i, d)$ um estado do modelo estocástico no grafo completo, então a partículas saltaram, $v=(d+a) / n$ é a proporção coberta, ou seja, o conjunto de vértices visitados, $1-v$ é proporção de partículas ainda inativas e $(1-v) n$ é número de partículas que permanecem inativas e em média $(1-v) a$ partículas saltaram e sobreviveram, isto é, acordaram partículas inativas. Seja $a=\beta n$, onde $\beta$ é a proporção de partículas ativas. A probabilidade de uma partícula inativa fixa ser acordada por uma das $(1-v) a$ partículas que saltam e sobrevivem é $1 /(1-v) n$. Logo

$$
\left(1-\frac{1}{(1-v) n}\right)^{(1-v) \beta n}
$$

é a probabilidade de uma partícula inativa fixa não ser despertada por nenhuma das $(1-v) a$ partículas que saltaram e sobreviveram, que tende a $\exp (-\beta)$ quando $n$ tende a infinito. Então acordam em média $(1-v) n(1-\exp (-\beta))$ partículas. 
Voltando ao modelo determinístico, temos que imediatamente após o instante $t+1$ as proporções, em média, de partículas mortas e ativas são, respectivamente,

$$
\begin{gathered}
d_{t+1}=d_{t}+\left(d_{t}+a_{t}\right) a_{t} \\
a_{t+1}=\left(1-d_{t}-a_{t}\right)\left(1-\exp \left(-a_{t}\right)\right)+\left(1-d_{t}-a_{t}\right) a_{t} \\
=\left(1-d_{t}-a_{t}\right)\left(1+a_{t}-\exp \left(-a_{t}\right)\right) .
\end{gathered}
$$

Isso nos diz que para os primeiros instantes de tempo $a_{t}$ quase dobrará seu valor, o que notamos através das simulações, pois $\exp \left(-a_{t}\right)$ é próximo de $1-a_{t}$ quando $a_{t}$ é pequeno. Observamos que, como $1-x<\exp (-x)<1-x+x^{2} / 2$, $x \in(0,1)$, temos que $a_{t+1}=\left(1-m_{t}-a_{t}\right)\left(1+a_{t}-\exp \left(-a_{t}\right)\right)<2 a_{t} \mathrm{e}$

$$
\begin{gathered}
\left(1-m_{t}-a_{t}\right)\left(a_{t}-\left(a_{t} / 2\right)^{2}\right)<\left(1-m_{t}-a_{t}\right)\left(1-\exp \left(-a_{t}\right)\right) \\
\Rightarrow\left(1-m_{t}-a_{t}\right)\left(2 a_{t}-\left(a_{t} / 2\right)^{2}\right)<\left(1-m_{t}-a_{t}\right)\left(1+a_{t}-\exp \left(-a_{t}\right)\right)=a_{t+1} .
\end{gathered}
$$

Logo, $\left(1-m_{t}-a_{t}\right) a_{t}\left(2-a_{t} / 2\right)<a_{t+1}<2 a_{t}$. Portanto, se $t<t_{1}$ e $0,001 \leq a_{t_{1}}<$ 0,002 valem as desiguladades

$$
1,994 a_{t}<(1-0,000006-0,002) 2 a_{t} \leq a_{t+1}<2 a_{t}
$$

onde 0,000006 provém do fato de que $d_{t}<0,003 a_{t}$, que será demonstrado posteriormente, e $a_{0} \leq a_{1} \leq \ldots \leq a_{t_{1}}$. Esses fatos serão úteis mais adiante na prova de nosso próximo teorema. 
Voltemos e observemos que $n$, o número de vértices, está fixo e que $t$ varia. Notemos que $v_{t}=d_{t}+a_{t}$, a proporção de vértices visitados com esta nova dinâmica, deveria ser denotada por $v_{t}(n)=d_{t}(n)+a_{t}(n)$. A Figura 3.1 mostra a evolução de $a_{t}$ e $v_{t}$ para $n=500,1000,2000$ e 5000, que comparamos com a Figura 2.1: as correlações não estragam o comportamento global do processo quando comparado com este no qual temos independência e trabalhamos com as médias.
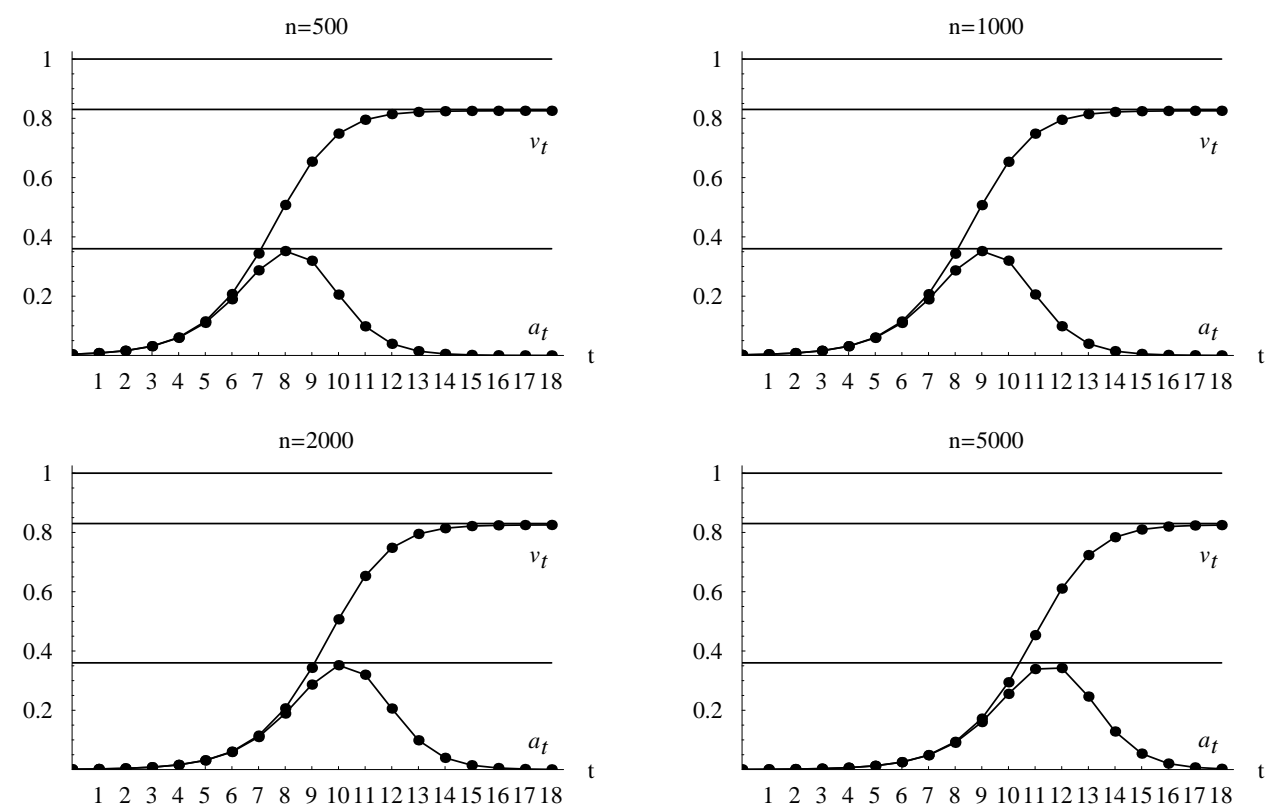

Figura 3.1: Evolução de $a_{t}$ e $v_{t}$ para $n=500,1000,2000$ e 5000. Linhas de 0,36 e 0,83 são indicadas. $\left(a_{0}=2 / n\right.$ e $\left.d_{0}=0\right)$.

Isso motiva nosso objetivo, demonstrado após os lemas 3.2 e 3.3:

Teorema $3.1 \quad$ (1) Seja $\lim _{t \rightarrow \infty} v_{t}=v=v(n)$. Então $v(n)$ pertence ao intervalo $(0,8248 ; 0,8261)$ para todo $n$. 
(2) Existe $a_{M}=a_{M}(n)$, o máximo dos $a_{t}$, e este máximo pertence ao intervalo $(0,3407 ; 0,3548)$ para todo $n$.

(3) $a_{M}$ se consegue num $t=t(n)$ que é da ordem de $\log n$ pois $\log (0,0005 n) / \log 2<M<\log (0,001 n) / \log 1,994+30$

Esses resultados capturam muito bem o que acontece nas simulações do grafo completo. Esclarecemos que $a_{0}$ deve ser pequeno pois no grafo completo sempre começamos com duas partículas ativas, logo a proporção inicial de partículas acordadas é $2 / n$. Porém podemos tomar $a_{0} \in(0,1)$ à vontade. Quando $a_{0}=2 / n$ e $d_{0}=0$ os cálculos computacionais indicam que $v$ é constante para todo $n$, $0,825455 \ldots$, isto é, que $\lim _{n \rightarrow \infty} \lim _{t \rightarrow \infty} v_{t}$ existe e que $a_{t}$ atinge um máximo, que varia $\operatorname{com} n$, no intervalo $(0,3407 ; 0,3548)$.

Os próximos dois lemas são fundamentais na demonstração do teorema 3.1.

Lema 3.2 (1) Existe $M=M(n)$ tal que

$$
a_{0} \leq a_{1} \leq \ldots \leq a_{M-1} \leq a_{M}>a_{M+1}>a_{M+2}>\ldots
$$

(2) $0,15 \leq a_{M}$

(3) $\lim _{t \rightarrow \infty} v_{t}=\lim _{t \rightarrow \infty} d_{t}=v=v(n)<1$.

(4) Se $a_{t} \leq 0,002$ então $d_{t}<0,003 a_{t}$.

O seguinte resultado é conseqüência imediata das fórmulas $a_{t+1}=\left(1-v_{t}\right)\left(1+a_{t}-\exp \left(-a_{t}\right)\right)$ e $d_{t+1}=d_{t}+\left(d_{t}+a_{t}\right) a_{t}$. Este lema nos diz 
como obter novos limitantes inferiores e superiores, a partir de limitantes dados, para $d_{t+1}$ e $a_{t+1}$ que independam de $n$.

Lema 3.3 Se $z<a_{t}<w$ e $y<d_{t}<u$, então

$$
\begin{gathered}
y+(y+z) z<d_{t+1}<u+(u+w) w \\
(1-w-u)(1+z-\exp (-z))<a_{t+1}<(1-z-y)(1+w+\exp (-w)) .
\end{gathered}
$$

A prova do teorema 3.1 baseia-se nos dois lemas anteriores. As simulações no grafo completo são nossa bússola, dizem-nos que a ação se desenrola em poucas unidades de tempo, menos do que 30 deles. A idéia é esta: como $0,15 \leq a_{M}$, existe $t_{1}<M$ para o qual $0,001<a_{t_{1}}<0,002\left(a_{t}<2 a_{t-1}\right)$. Agora dividimos o segmento $(0,001 ; 0,002)$ em segmentos de comprimento muito pequeno e fazemos, para cada um desses subintervalos, com que $z$ e $w$ tomem os valores extremos desses subintervalos, com $y=0$ e $u=0,000006$. Em seguida, aplicamos o lema anterior a fim de obtermos novos valores para $z, w, y$ e $u$ para cada subintervalo de $(0,001 ; 0.002)$. Com isto obteremos cotas, dependentes de cada segmento, para $a_{t_{1}+1}, a_{t_{1}+2}, \ldots, a_{t_{1}+30}$ e para $d_{t_{1}+1}, d_{t_{1}+2}, \ldots, d_{t_{1}+30}$ que são suficientes para encontrarmos um intervalo no qual $\lim _{t \rightarrow \infty} d_{t}=v$ está contido. Após feitos os cálculos para todo subintervalo de $(0,001 ; 0,002)$, temos pares de limitantes inferior e superior para $v$ que dependem de cada subintervalo. Basta agora tomarmos como limitante inferior de $v$ o mínimo entre todos eles após 30 iterações e como limitante superior de $v$ o máximo entre todos limitantes superiores depois de 30 
iterações.

Demonstração do teorema 3.1

Prova: (3): Dado que $a_{M} \geq 0,15$, existe $t_{1}<M$ tal que $0,001<a_{t_{1}}<0,002$. Mas se $t<t_{1}$, então $1,994 a_{t} \leq a_{t+1}<2 a_{t}$. Como $a_{0}=2 / n$, teremos que $0,001<a_{t_{1}}<2 / n 2^{t_{1}}$ e $2 / n 1,994^{t_{1}}<a_{t_{1}}<0,002$. Logo $t_{1}$ é da ordem de $\log n$.

Antes de demonstrarmos (1) e (2), vejamos como foram calculados as cotas para $a_{t}$ e $d_{t}$ conforme o lema 3.3:

Sejam $h \in\{5,6, \ldots\}$ e $r=1 / 10^{h}$. Teremos então $(0,002-0,001) / r$ subintervalos. Escrevemos $z=0.001+(k-1) r, w=0.001+k r, k \in\{1,2, \ldots ; 0,001 / r\}$, com $y=0$ e $u=0,000006$. Logo podemos calcular novos limitantes para $a_{t+1} \mathrm{e}$ $d_{t+1}$ e assim sucessivamente.

(2): Um cálculo computacional mostra-nos que 30 iterações, para $h=8$ e todo subintervalo de $(0,001 ; 0.002)$, são suficientes para afirmar que o máximo se dá em algum $t \in\left\{t_{1}+1, t_{1}+2, \ldots, t_{1}+30\right\}$ e pertence ao intervalo $(0,3407 ; 0,3548)$. Afirmamos também que este máximo ocorre em $t_{1}+10$ ou $t_{1}+11$, dependendo de cada subintervalo, o que em qualquer caso é de ordem $\log n$.

(1): Observemos que $d_{t}<d_{t+1} \mathrm{e}$

$$
d_{t+1}=d_{t}+\left(d_{t}+a_{t}\right) a_{t}<d_{t}+a_{t} \Rightarrow d_{t+i}<d_{t}+\sum_{j=t}^{i-1} a_{j} .
$$

$\operatorname{Logo} d_{t_{1}+30}<\lim _{t \rightarrow \infty} d_{t} \leq d_{t_{1}+30}+\sum_{i=t_{1}+30}^{\infty} a_{i}$. Como $a_{t_{1}+30}<7.10^{-9}$ se $h=8$ e para $M+1$ observamos $v_{M+1}>0,60$, resultado este calculado computacionalmente, temos $a_{t+1}<0,8 a_{t}$ para $t>M$, e o resultado é obtido pois 
$d_{t_{1}+30} \in(0,8248 ; 0.82609)$ e $\sum_{i=t_{1}+30}^{\infty} a_{i}<5 a_{t_{1}+30}$.

Agora começamos as provas dos lemas 3.2 e 3.3. Um argumento probabilístico é válido para o seguinte lema.

Lema 3.4 Para todo $t \in\{0,1,2, \ldots\}$ temos que $a_{t}>0, d_{t} \geq 0$ e $0<v_{t}<1$ para todo $n$.

Prova : $a_{0}>0, d_{0}=0$ e $v_{0}=a_{0}$. Suponhamos que $a_{t}>0, d_{t} \geq 0$ e $0<v_{t}<1$, então $d_{t+1}=d_{t}+\left(d_{t}+a_{t}\right) a_{t}>0$.

Agora $0<a_{t} \leq d_{t}+a_{t}=v_{t}=1-x<1$, para algum $x>0$, logo

$$
\begin{aligned}
v_{t+1}= & d_{t+1}+a_{t+1} \\
& =d_{t}+a_{t}+\left(1-d_{t}-a_{t}\right)\left(1-\exp \left(-a_{t}\right)\right) \\
& =1-x+\left(1-d_{t}-a_{t}\right)\left(1-\exp \left(-a_{t}\right)\right) \\
& <1-x+x \\
& =1 .
\end{aligned}
$$

Também $a_{t+1}=\left(1-v_{t}\right)\left(1+a_{t}-\exp \left(-a_{t}\right)\right)>0$

Como corolário do lema anterior temos o seguinte: $\lim _{t \rightarrow \infty} v_{t}=v$ (existe para cada $n$ ) pois $0<v_{t}<1$ e $v_{t}<v_{t+1}$. Além disso, $0 \leq d_{t}<v_{t}<1$ e $d_{t}<d_{t+1}$, logo $\lim _{t \rightarrow \infty} d_{t}=d($ existe para cada $n)$

Nosso primeiro resultado importante na prova dos Lemas 3.2 e 3.3 é conseqüência imediata do fato de que $\lim _{t \rightarrow \infty} d_{t}$ existe. 
Lema $3.5 \lim _{t \rightarrow \infty} a_{t}=0$.

Prova : Suponhamos que exista uma subseqüência infinita $\left\{t_{k}\right\}$ tal que, $\forall t_{k}$, $a_{t_{k}}>\epsilon$, então $d_{t_{k}+1}=d_{t_{k}}+\left(d_{t_{k}}+a_{t_{k}}\right) a_{t_{k}}$, o que implica que $d_{t_{k}+1}>d_{t_{k}}+\left(d_{t_{k}}+\epsilon\right) \epsilon$. Portanto teríamos que $d \geq d+(d+\epsilon) \epsilon$, o que é absurdo. Logo tal subseqüência não existe e $\lim _{t \rightarrow \infty} a_{t}=0$.

Portanto, $\lim _{t \rightarrow \infty} d_{t}=v$ e existe $t_{0}$ tal que $a_{t_{0}}>a_{t_{0}+1}$, pois a seqüência dos $a_{t}$ não pode ser crescente. Seja $M$ o primeiro $t$ tal que $a_{t}>a_{t+1}$. Agora observemos que $g(x)=1+x-\exp (-x)$ é crescente em $(0,1)$, já que $g^{\prime}(x)>0$. Esses fatos serão usados no próximo resultado.

Lema $3.6 a_{M}>a_{M+1}>a_{M+2}>\ldots$

\section{Prova :}

$$
\begin{aligned}
a_{M+2} & =\left(1-v_{M+1}\right)\left(1+a_{M+1}-\exp \left(-a_{M+1}\right)\right) \\
& <\left(1-v_{M}\right)\left(1+a_{M+1}-\exp \left(-a_{M+1}\right)\right) \\
& <\left(1-v_{M}\right)\left(1+a_{M}-\exp \left(-a_{M}\right)\right) \\
& =a_{M+1}, \text { e os outros casos seguem por indução. }
\end{aligned}
$$

Portanto, $v \geq 1 / 2$, pois $\left(1-d_{t}-a_{t}\right) a_{t}\left(2-a_{t} / 2\right)<a_{t+1}$, o que implica que $\lim _{t \rightarrow \infty}\left(1-d_{t}-a_{t}\right)\left(2-a_{t} / 2\right)=(1-v) 2 \leq 1 \Leftrightarrow 1 / 2<v$. Ademais, $v_{M-1}<1 / 2$ pois $a_{M-1} \leq a_{M}<\left(1-v_{M-1}\right) 2 a_{M-1}$. 
A seguir vamos obter um limitante infeior para $d_{t+1}$ que nos será útil para obter um limitante para $a_{M}$.

\section{Lema 3.7}

$$
\begin{aligned}
& d_{t+1}=\left(a_{t}\right)^{2} \\
& \left(a_{t-1}\right)^{2}\left(1+a_{t}\right) \quad+ \\
& \left(a_{t-2}\right)^{2}\left(1+a_{t-1}\right)\left(1+a_{t}\right) \quad+ \\
& \left(a_{0}\right)^{2}\left(1+a_{1}\right) \ldots\left(1+a_{t}\right) .
\end{aligned}
$$

\section{Prova :}

$$
\begin{aligned}
d_{t+1}=d_{t}+\left(d_{t}+a_{t}\right) a_{t} & \\
& =d_{t}\left(1+a_{t}\right)+\left(a_{t}\right)^{2} \\
& =\left(d_{t-1}\left(1+a_{t-1}\right)+\left(a_{t-1}\right)^{2}\right)\left(1+a_{t}\right)+\left(a_{t}\right)^{2} \\
& =d_{t-1}\left(1+a_{t-1}\right)\left(1+a_{t}\right)+\left(a_{t-1}\right)^{2}\left(1+a_{t}\right)+\left(a_{t}\right)^{2} \\
& =\left(a_{t}\right)^{2}+\left(a_{t-1}\right)^{2}\left(1+a_{t}\right)+\left(a_{t-2}\right)^{2}\left(1+a_{t-1}\right)\left(1+a_{t}\right)+\ldots \\
& +\left(a_{0}\right)^{2}\left(1+a_{1}\right) \ldots\left(1+a_{t}\right)
\end{aligned}
$$

$\operatorname{Logo} d_{t+1}>\sum_{i=0}^{t}\left(a_{i}\right)^{2}$. Como $a_{t}<2 a_{t-1}$, temos que $a_{t}<2^{i} a_{t-i}$. Daí $d_{t}>a_{t}^{2} / 2^{2 t}+a_{t}^{2} / 2^{2(t-1)}+\ldots+a_{t}^{2} / 2^{2}>a_{t}^{2}(1 / 4+1 / 16)$. Portanto, $d_{t}>5 / 16\left(a_{t}\right)^{2}$. 
Agora será fácil mostrar, usando o resultado anterior, que $a_{M}<0,41$, pois $0<g(x)=\left(1-x-5 / 16 x^{2}\right)(1+x-\exp (-x))<0,41$ no intervalo $(0 ; 0,41)$.

Lema $3.8 a_{t}<0,41$.

Prova : Tomemos $a_{0}<0,41\left(a_{0}=2 / n\right)$. Agora suponhamos $a_{t}<0,41$, logo

$$
\begin{aligned}
a_{t+1} & =\left(1-v_{t}\right)\left(1+a_{t}-\exp \left(-a_{t}\right)\right) \\
& <\left(1-a_{t}-5 / 16\left(a_{t}\right)^{2}\right)\left(1+a_{t}-\exp \left(-a_{t}\right)\right) \\
& <0,41
\end{aligned}
$$

Vimos que $a_{M}<0,41$, e agora saberemos que $0,15 \leq a_{M}<0,41$. Este resultado nos diz que, para cada $n$, a seqüência $\left\{a_{t}\right\}$ passa pelo intervalo $(0,001 ; 0,002)$.

Lema $3.9 a_{M} \geq 0,15$.

Prova : Suponhamos $a_{M}<0,15$ e vejamos que $d_{t}<a_{t}$ para todo $t$. Primeiramente sabemos que $a_{0}>m_{0}$. Suponhamos agora que $a_{t}>m_{t}$. Como $d_{t+1}<a_{t}\left(1+2 a_{t}\right)<1,3 a_{t}$ e $a_{t+1}>\left(1-d_{t}-a_{t}\right) a_{t}\left(2-a_{t} / 2\right)>0,7 a_{t}(2-0,15 / 2)>$ $1,3 a_{t}$, então $d_{t+1}<a_{t+1}$. Porém isto contradiz o fato de que $\lim _{t \rightarrow \infty} d_{t}=v$ e $\lim _{t \rightarrow \infty} a_{t}=0$. Portanto, $a_{M} \geq 0,15$.

Já obtivemos um limitante inferior para $d_{t}$, agora obteremos um limitante superior para $d_{t}$ em termos de $a_{t}$. 
Lema 3.10 Se $a_{t} \leq 0,002$ então $d_{t}<0,003 a_{t}$.

Prova : Inicialmente, temos que $d_{0}<0,003 a_{0}$. Suponhamos que $d_{t}<0,003 a_{t}$. Logo $0,003 a_{t+1}-d_{t+1}>0,003\left(1-0,003 a_{t}-a_{t}\right)\left(1+a_{t}-\exp -a_{t}\right)-\left(0,003 a_{t}+\left(0,003 a_{t}+a_{t}\right) a_{t}\right)$ $=0,003\left(1-0,003 a_{t}-a_{t}\right)\left(1+a_{t}-\exp -a_{t}\right)-a_{t}\left(0,003+(0,003+1) a_{t}\right)>0$, para $a_{t} \leq 0,002$. Portanto, $0,003 a_{t}-d_{t}>0$.

\subsection{Duas vidas por partícula}

Similarmente à seção anterior, podemos definir as equações de campo médio para o caso em que cada partícula possui 2 vidas. Uma diferença que podemos notar inicialmente diz respeito à percentagem de partículas mortas: de um instante a outro ela só pode ser acrescida das particulas ativas com uma vida $\left(a_{t}^{1}\right)$ que morreram, pois as partículas ativas com duas vidas $\left(a_{t}^{2}\right)$ não podem perder 2 numa única passagem. Daí

$$
d_{t+1}=d_{t}+\left(d_{t}+a_{t}\right) a_{t}^{1}
$$

Da mesma maneira, a equação que rege as partículas inativas é dada por $i_{t+1}=i_{t}-i_{t}\left(1-\exp \left(-a_{t}\right)\right)=i_{t} \exp \left(-a_{t}\right)$, onde aqui $a_{t}=a_{t}^{1}+a_{t}^{2}$. Logo

$$
a_{t+1}=1-d_{t+1}-i_{t+1}=\left(1-d_{t}-a_{t}\right)\left(1+a_{t}-\exp \left(-a_{t}\right)\right)+\left(d_{t}+a_{t}\right) a_{t}^{2} .
$$


Além disso, escrevemos as partículas ativas com uma vida da seguinte forma: $a_{t+1}^{1}=\left(1-d_{t}-a_{t}\right) a_{t}^{1}+\left(d_{t}+a_{t}\right) a_{t}^{2}$, isto é, a soma das partículas ativas com uma vida que sobreviveram mais as partículas ativas com duas vidas que perderam uma vida. Logo $a_{t+1}^{2}=\left(1-d_{t}-a_{t}\right) a_{t}^{2}+\left(1-d_{t}-a_{t}\right)\left(1-\exp \left(-a_{t}\right)\right)$, o que é coerente, pois temos a adição das partículas ativas com duas vidas que permaneceram com duas vidas mais as partículas que foram acordadas. Também para 2 vidas por partícula a fórmula para $a_{t}$ mostra-nos, conforme observamos nos cálculos computacionais, que para os primeiros instantes de tempo $a_{t}$ quase dobrará seu valor, pois $\exp \left(-a_{t}\right)$ é próximo de $1-a_{t}$ quando $a_{t}$ é pequeno. Dado que $1-\exp (-x)<x, x \in(0,1)$, lembramos que $a_{t+1}=\left(1-v_{t}\right)\left(1+a_{t}-\exp \left(-a_{t}\right)\right)+v_{t} a_{t}^{2}<\left(1-v_{t}\right) 2 a_{t}+v_{t} a_{t}^{2}=2 a_{t}-v_{t}\left(2 a_{t}-a_{t}^{2}\right)<2 a_{t}$.

Definimos $f_{1}(t)=\left(1-d_{t}-a_{t}\right)\left(1+a_{t}-\exp \left(-a_{t}\right)\right)$, a fração de partículas ativas no modelo com uma vida por partícula, e $f_{2}(t)=\left(1-d_{t}-a_{t}\right)\left(1+a_{t}-\right.$ $\left.\exp \left(-a_{t}\right)\right)+\left(d_{t}+a_{t}\right) a_{t}^{2}$, a porção de ativas no modelo com 2 vidas, para todo $t$. Como $a_{t+1}<2 a_{t}$ e $f_{2}(t) \geq f_{1}(t)$, existe $t_{2} \in N$ tal que $f_{2}\left(t_{2}\right) \in(0,001 ; 0,002)$. Isso somado à afirmação de que $d_{t}<0,005 a_{t}$ se $a_{t} \leq 0,004$, que é demonstrada abaixo, são fatos usados na demostração do teorema seguinte.

Novamente, notemos que $n$, o número de vértices, está fixo e que $t$ varia. Aqui também definimos $v_{t}=d_{t}+a_{t}$, a proporção de vértices visitados com esta nova dinâmica. A figura 3.2 mostra a evolução de $a_{t}$ e $v_{t}$ para $n=500,1000,2000 \mathrm{e}$ 5000, que comparamos com a figura 2.2: no modelo estocástico e no campo médio 
os gráficos se comportam muito parecidamente, apesar de as correlações não serem levadas em conta no modelo determinístico. A demonstração do teorema subsequente será apresentada abaixo dos lemas 3.12 e 3.13 .
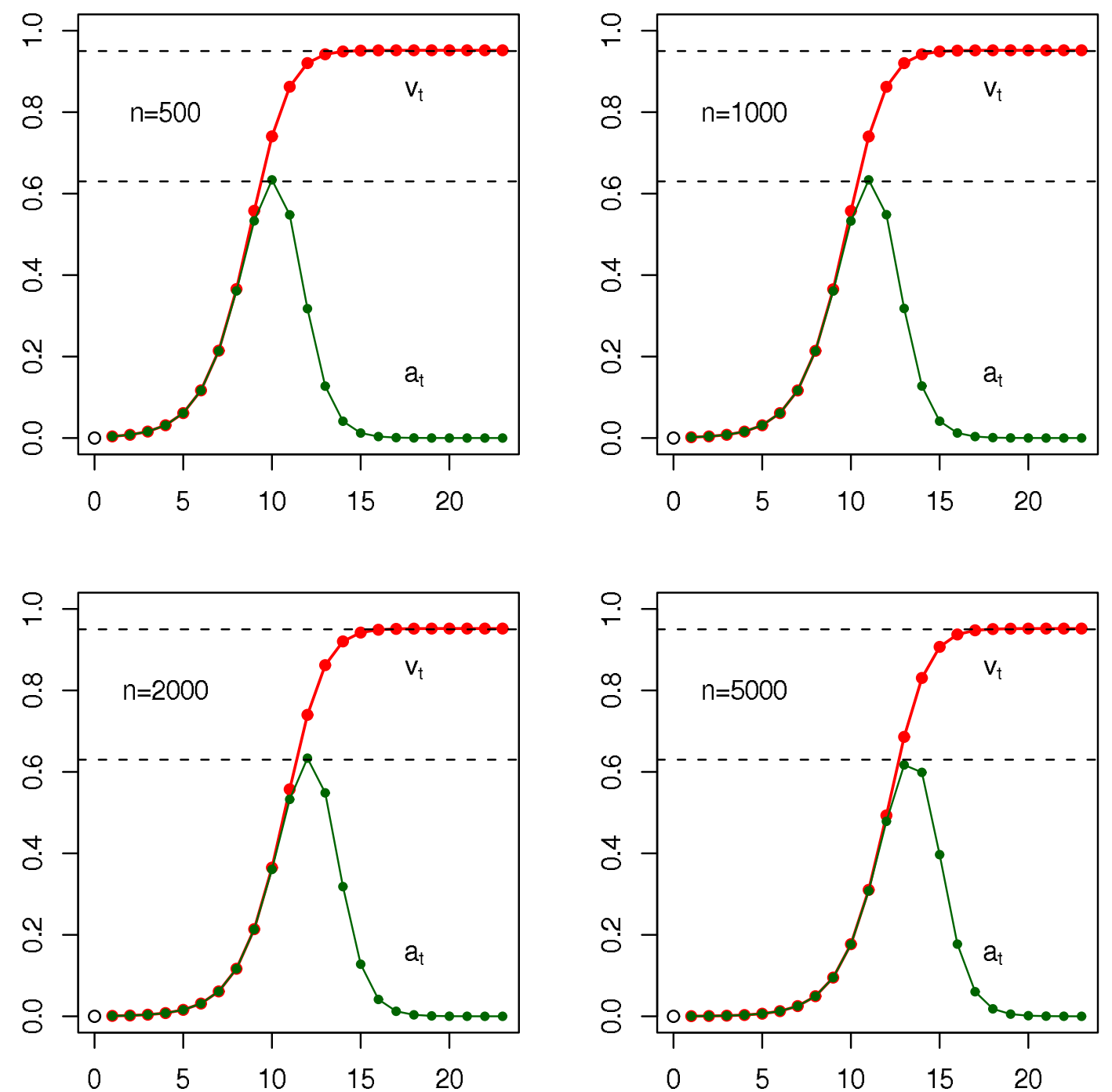

Figura 3.2: Evolução de $a_{t}$ e $v_{t}$ para $n=500,1000,2000$ e $5000\left(a_{0}=2 / n\right.$ e $\left.d_{0}=0\right)$. Linhas de 0,63 e 0,95 são indicadas.

Teorema 3.11 (1) Existe $\lim _{t \rightarrow \infty} v_{t}=\lim _{t \rightarrow \infty} d_{t}=v=v(n)$. 
(2) Existe $a_{M^{\prime}}=a_{M^{\prime}}(n)$, o máximo dos $a_{t}$, e este máximo pertence ao intervalo $(0,594 ; 0,65)$ para todo $n$.

O intervalo $(0,594 ; 0,65)$ para $a_{M^{\prime}}$ retrata muito bem o que ocorre nas simulações do grafo completo para 2 vidas por partícula. Assim como no caso em que há apenas uma vida por partícula, tomamos $a_{0}$ pequeno, mais precisamente $2 / n$, pois sempre começamos com duas partículas ativas, todavia podemos tomar $a_{0} \in(0,1)$ à vontade. A despeito de não apresentarmos um intervalo para $\lim _{t \rightarrow \infty} v_{t}=v=v(n)$, faz-se necessário enfatizar que os cálculos computacionais indicam que $v$ é constante para todo $n$ e tende a $0,9519026 \ldots$. A dificuldade em encontrar um intervalo que contenha $v$ está nas cotas superiores $w$ e $u$ para $d_{t}$ e $a_{t}$ : os cálculos computacionais indicam que $w+u$ supera 1 após alguns instantes de tempo, o que compromete o cálculo de todas os limitantes posteriormente, como podemos ver no lema 3.13. Os dois lemas adjacentes são essenciais na demonstração do teorema 3.11 .

Lema 3.12 (1) $0,15 \leq a_{M^{\prime}}$

(2) Se $a_{t} \leq 0,004$ então $d_{t}<0,005 a_{t}$.

\section{Prova :}

(1): Usando $f_{2}(t)$ e $f_{1}(t)$, citados no início desta seção, como as proporções de partículas ativas com 1 e 2 vidas, respecivamente, e considerando a desigualdade $f_{2}(t) \geq f_{1}(t)$, temos que $f_{2}\left(M^{\prime}\right) \geq f_{2}(M) \geq f_{1}(M) \geq 0,15$. Logo, $a_{M^{\prime}} \geq 0,15$. 
(2): O limitante superior para $d_{t}$ é fundamental para fazermos os cálculos computacionais, já que é a partir dessa cota que os efetuamos.Inicialmente, temos que $d_{0}<0,005 a_{0}$. Suponhamos que $d_{t}<0,005 a_{t}$. Logo $0,005 a_{t+1}-d_{t+1}>0,005\left(1-0,005 a_{t}-a_{t}\right)\left(1+a_{t}-\exp -a_{t}\right)-\left(0,005 a_{t}+\left(0,005 a_{t}+a_{t}\right) a_{t}\right)$ $=0,005\left(1-0,005 a_{t}-a_{t}\right)\left(1+a_{t}-\exp -a_{t}\right)-a_{t}\left(0,005+(0,005+1) a_{t}\right)>0$, para $a_{t} \leq 0,004$. Portanto, $0,005 a_{t}-d_{t}>0$.

O lema que se segue é conseqüência imediata das expressões $a_{t+1}=\left(1-v_{t}\right)\left(1+a_{t}-\exp \left(-a_{t}\right)\right)+v_{t} a_{t}^{2}, a_{t+1}^{1}=\left(1-v_{t}\right) a_{t}^{1}+v_{t} a_{t}^{2}$, $a_{t+1}^{2}=\left(1-v_{t}\right)\left(1+a_{t}^{2}-\exp \left(-a_{t}\right)\right)$ e $d_{t+1}=d_{t}+\left(d_{t}+a_{t}\right) a_{t}^{1}$. Diz-nos como obter novos limitantes inferiores e superiores, a partir de limitantes dados, para $d_{t+1}, a_{t+1}, a_{t+1}^{1}$ e $a_{t+1}^{2}$ que independam de $n$.

Lema 3.13 Se $z<a_{t}<w, z_{1}<a_{t}^{1}<w_{1}, z_{2}<a_{t}^{2}<w_{2}$ e $y<d_{t}<u$, então

$$
\begin{gathered}
y+(y+z) z_{1}<d_{t+1}<u+(u+w) w_{1} \\
(1-w-u)(1+z-\exp (-z))+(y+z) z_{2}<a_{t+1}<(1-z-y)(1+w+\exp (-w))+(u+w) w_{2}, \\
(1-u-w) z_{1}+(z+y) z_{2}<a_{t+1}^{1}<(1-z-y) w_{1}+(w+u) w_{2}, \\
(1-w-u)\left(1+z_{2}-\exp (-z)\right)<a_{t+1}^{2}<(1-z-y)\left(1+w_{2}+\exp (-w)\right) .
\end{gathered}
$$

Antes de apresentarmos a prova de 3.11, comentaremos como se chegou a ela. Seguindo os mesmos passos do casos anterior, com o acréscimo da dificuldade 
em encontrar cotas para $z_{1}, w_{1}, z_{2}, w_{2}$ no instante inicial, temos $0,15 \leq a_{M^{\prime}}$ e, portanto, $t_{2}<M^{\prime}$ para o qual $0,001<a_{t_{2}}<0,002\left(a_{t}<2 a_{t-1}\right)$. Em seguida, subdividimos o segmento $(0,001 ; 0,002)$ em subintervalos de comprimento muito pequeno e fazemos, para cada um desses subintervalos, com que $z$ e $w$ tomem os valores extremos desses subintervalos, com $y=0$ e $u=0,00001$. Para obtermos cotas para $z_{1}, w_{1}, z_{2}, w_{2}$ procedemos da seguinte forma: $a_{t_{2}-1} \geq z / 2$, onde $z=$ $0.001+(k-1) r, w=0.001+k r, h \in\{5,6, \ldots\}$ e $r=1 / 10^{h}$. Logo $a_{t_{2}}^{1}=$ $\left(1-2 v_{t_{2}-1}\right) a_{t_{2}-1}^{1}+v_{t_{2}-1} a_{t_{2}-1}>(z / 2)^{2}=z_{1}$. Daí $w>a_{t_{2}-1}+z_{1}$ e $w_{2}=w-z_{1}$. Similarmente, $a_{t_{2}}^{1}=\left(1-2 v_{t_{2}-1}\right) a_{t_{2}-1}^{1}+v_{t_{2}-1} a_{t_{2}-1}<\left(1-2 v_{t_{2}-1}\right) z+v_{t_{2}-1} z=$ $\left(1-v_{t_{2}-1}\right) z<(1-z / 2) z=w_{1}$. Logo $a_{t_{2}}^{2}>z-w_{1}=z_{2}$. Posteriromente, aplicamos o lema anterior a fim de obtermos novos valores para $z, z_{1}, z_{2}, w, w_{1}, w_{2}, y, u$ para cada subintervalo de $(0,001 ; 0.002)$. Com isto obteremos cotas, dependentes de cada segmento, para $a_{t_{1}+1}, a_{t_{1}+2}, \ldots, a_{t_{1}+20}$ e para $d_{t_{1}+1}, d_{t_{1}+2}, \ldots, d_{t_{1}+20}$ que são suficientes para encontrarmos um intervalo no qual $a_{M^{\prime}}=a_{M^{\prime}}(n)$ está contido.

Demonstração do teorema 3.11

\section{Prova :}

(1): Ver lemas 3.14 e 3.15 logo abaixo, os quais fornecem um panorama mais detalhado desse item.

(2): Um cálculo computacional mostra-nos que 20 iterações, para $h=8$ e todo subintervalo de $(0,001 ; 0.002)$, são suficientes para afirmar que o máximo se dá em algum $t \in\left\{t_{1}+1, t_{1}+2, \ldots, t_{1}+20\right\}$ e pertence ao intervalo $(0,594 ; 0,65)$. 
Para cada subintervalo, temos pares de limitantes inferior e superior para $a_{M^{\prime}}$. Agora tomamos como limitante inferior de $a_{M^{\prime}}$ o mínimo entre todos eles após 20 iterações e como limitante superior de $a_{M^{\prime}}$ o máximo entre todos limitantes superiores depois de 20 iterações, o que ocorre, afirmamos, para $t_{2}+11$ ou $t_{2}+12$, dependendo de cada subintervalo de $(0,001 ; 0.002)$.

Lema 3.14 Para todo $t \in\{0,1,2, \ldots\}$ temos que $a_{t}>0, d_{t} \geq 0$ e $0<v_{t}<1$ para todo $n$.

Prova : $a_{0}>0, d_{0}=0$ e $v_{0}=a_{0}$. Suponhamos que $a_{t}>0, d_{t} \geq 0$ e $0<v_{t}<1$, então $d_{t+1}=d_{t}+\left(d_{t}+a_{t}\right) a_{t}^{1} \geq 0$.

Temos $0<a_{t} \leq d_{t}+a_{t}=v_{t}=1-x<1$, para algum $x>0, \log 0$

$$
\begin{aligned}
v_{t+1}= & d_{t+1}+a_{t+1} \\
& =d_{t}+v_{t} a_{t}+\left(1-d_{t}-a_{t}\right)\left(1+a_{t}-\exp \left(-a_{t}\right)\right) \\
& =d_{t}+a_{t}+\left(1-d_{t}-a_{t}\right)\left(1-\exp \left(-a_{t}\right)\right) \\
& =1-x+\left(1-d_{t}-a_{t}\right)\left(1-\exp \left(-a_{t}\right)\right)<1-x+x \\
& =1 .
\end{aligned}
$$

$\mathrm{E} a_{t+1}=\left(1-v_{t}\right)\left(1+a_{t}-\exp \left(-a_{t}\right)\right)+v_{t} a_{t}^{2}>0$

Uma das implicações do lema anterior nos diz que $\lim _{t \rightarrow \infty} v_{t}=v$ existe para cada $n$, pois $0<v_{t}<1$ e $v_{t}<v_{t+1}$. Além disso, $0 \leq d_{t}<v_{t}<1$ e $d_{t} \leq d_{t+1}$, então $\lim _{t \rightarrow \infty} d_{t}=d$ existe para cada $n$. 
O resultado abaixo é capital na prova do teorema 3.11 e conseqüência direta do fato de que $\lim _{t \rightarrow \infty} d_{t}$ existe.

Lema $3.15 \lim _{t \rightarrow \infty} a_{t}=0$.

Prova : Suponhamos que exista uma subseqüência infinita $\left\{t_{k}\right\}$ tal que, $\forall t_{k}$, $a_{t_{k}}^{1}>\epsilon$, então $d_{t_{k}+1}=d_{t_{k}}+\left(d_{t_{k}}+a_{t_{k}}\right) a_{t_{k}}^{1}$, o que implica que $d_{t_{k}+1}>d_{t_{k}}+$ $\left(d_{t_{k}}+\epsilon\right) \epsilon$. Portanto teríamos que $d \geq d+(d+\epsilon) \epsilon$, o que é absurdo. Logo tal subseqüência não existe e $\lim _{t \rightarrow \infty} a_{t}^{1}=0$. Provemos que $\lim _{t \rightarrow \infty} a_{t}$ existe: $v-d=\lim _{t \rightarrow \infty} v_{t}-\lim _{t \rightarrow \infty} d_{t}=\lim _{t \rightarrow \infty} a_{t}:=a . \quad$ Logo $\lim _{t \rightarrow \infty} a_{t}^{2}$ existe. Da expressão $a_{t+1}^{1}=\left(1-v_{t}\right) a_{t}^{1}+v_{t} a_{t}^{2}$ obtemos que $0=a^{1}=(1-v) 0+v a^{2}$. Logo $a^{2}=0$. Portanto, $\lim _{t \rightarrow \infty} a_{t}=0$.

\subsection{L vidas por partícula}

Analogamente aos casos anteriores, apresentamos as equações de campo médio para $L>2: d_{t+1}=d_{t}+v_{t} a_{t}^{1}, i_{t+1}=i_{t}-i_{t}\left(1-\exp \left(-a_{t}\right)\right)=i_{t} \exp \left(-a_{t}\right)$, onde $a_{t}=\sum_{i=1}^{L} a_{t}^{i}$. Logo

$$
a_{t+1}=1-d_{t+1}-i_{t+1}=\left(1-v_{t}\right)\left(1+a_{t}-\exp \left(-a_{t}\right)\right)+v_{t}\left(\sum_{i=2}^{L} a_{t}^{i}\right) .
$$

Além disso, $a_{t+1}^{i}=\left(1-v_{t}\right) a_{t}^{i}+v_{t} a_{t}^{i+1}, i \in\{1, \ldots, L-1\}$ e $a_{t+1}^{L}=\left(1-v_{t}\right) a_{t}^{L}+$ $\left(1-v_{t}\right)\left(1-\exp \left(-a_{t}\right)\right)$

Não estudamos o comportamento das equações de campo médio para $L \geq 3$, porém apresentamos abaixo um gráfico de $v$ pelo número de vidas por partícula 
para $n=15000$ vértices.

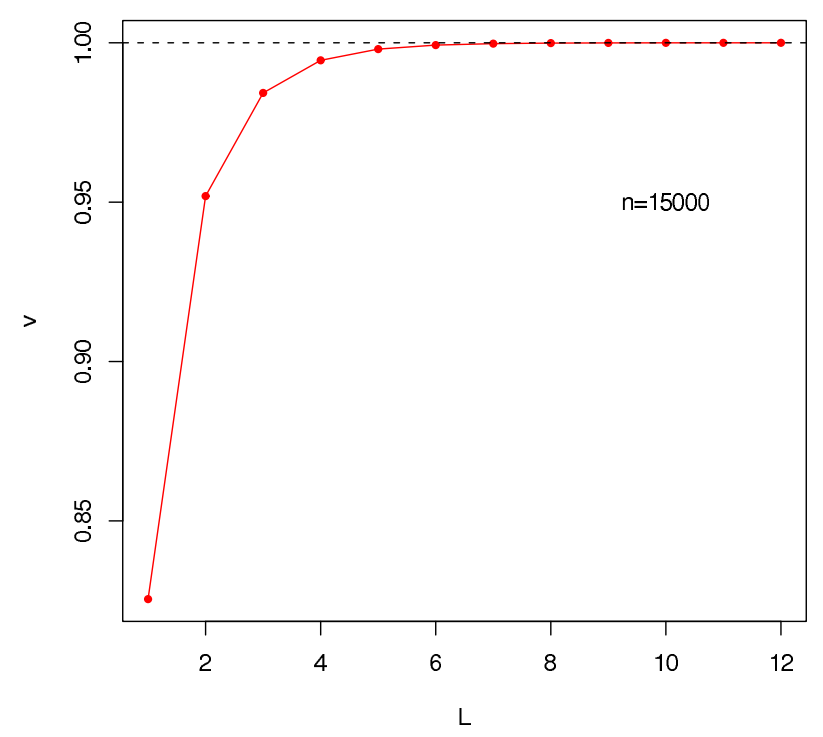

Figura 3.3: Cobertura do grafo completo para diferentes valores de $L$. Linha de 1,00 é indicada.

Conforme mostra-nos a figura, a cobertura do grafo $v$ parece depender exponencialmente de $L$, o número de vidas por partícula, o que não foi demonstrado neste trabalho. No entanto, tal dependência pode ser observada explícitamente em [7], trabalho que aborda passeios aleatórios a tempo contínuo, e a apresentamos abaixo para o caso em que há uma partícula por vértice:

$$
v=1-\exp \left(- \text { Lambert } W\left(-c e^{-c}\right)-c\right),
$$

onde $c=L+1$ e Lambert $W$ é a função inversa de $x \rightarrow x \exp (x)$. 


\section{Referências Bibliográficas}

[1] Alves, O. S. M., Machado, F. P., Popov, S. Yu. Phase transition for the frog model. Electron. J. Probab. 7 (16), 21 p., 2002.

[2] Alves, O. S. M., Machado, F. P., Popov, S. Yu. The shape theorem for the frog model. Ann. Appl. Probab. 12 (2), 533-546, 2002.

[3] Alves, O. S. M.,Lebensztayn, E., Machado, F. P., Martinez, M.Z. Random walks systems on complete graphs. Bulletin of the Brazilian Mathematical Society 37 (.), 571-580, 2006.

[4] Feller, W. An introduction to probability theory and its applications, Volume I. 3rd. ed., John Wiley \& Sons, New York, 1968.

[5] Lebensztayn, E., Machado, F. P., Popov, S. An improved upper bound for the critical probability of the frog model on homogeneous trees. J. Statist. Phys. 119 (1-2), 331-345, 2005.

[6] Lebensztayn, E., Machado, F. P., Martinez, M.Z. Random walks systems on $\mathbb{Z}$, Submetido, 2006.

[7] Leichsenring, A.R. Teoremas limite para um modelo epidêmico no grafo completo. Tese (Doutorado em Estatística) - Instituto de Matemática e Estatística, Universidade de São Paulo, São Paulo, 2007.

[8] Lima, E. L. Curso de análise, Volume 1. 11. ed., Associação Instituto Nacional de Matemática Pura e Aplicada, Rio de Janeiro, 2004. (Projeto Euclides). 